\title{
IL-6 mediates the signal pathway of JAK-STAT3-VEGF-C promoting growth, invasion and lymphangiogenesis in gastric cancer
}

\author{
GUIBIN ZHAO ${ }^{1}$, GUANGWEI ZHU ${ }^{2,3}$, YONGJIAN HUANG ${ }^{2,3}$, WEI ZHENG ${ }^{2,3}$, \\ JIN HUA $^{2,3}$, SHUGANG YANG ${ }^{2,3}$, JINFU ZHUANG ${ }^{2,3}$ and JIANXIN YE ${ }^{2,3}$ \\ ${ }^{1}$ Department of Oncology Surgery, Mindong Hospital Affiliated to Fujian Medical University, Fuan, Fujian; \\ ${ }^{2}$ Department of Gastrointestinal Surgery, First Hospital Affiliated to Fujian Medical University, Fuzhou, Fujian; \\ ${ }^{3}$ Key Laboratory of Ministry of Education for Gastrointestinal Cancer, Fujian Medical University, Fuzhou, Fujian, P.R. China
}

Received November 2, 2015; Accepted December 11, 2015

DOI: 10.3892/or.2016.4544

\begin{abstract}
Gastric cancer shows the highest invasive and metastasis features, especially lymph metastasis, which is closely associated with poor prognosis of gastric cancer. Although there is evidence that interleukin-6 (IL-6) can promote gastric cancer progression, the underlying specific mechanisms and the mechanisms of gastric cancer lymphangiogenesis are largely unknown. In the present study, we explore whether IL- 6 could promote the proliferation and invasion activity of gastric cancer cells, and whether IL-6 mediating VEGF-C production affected the lymphangiogenesis in gastric cancer cells. Our results revealed that IL-6 and its receptors (IL-6 and gp130) are broadly expressed in various gastric cancer cell lines including SGC-7901, MGC, MKN-28 and AGS. Exogenous IL-6 increased the ability of gastric cancer cell proliferation and invasion, which could be weakened by AG490. In addition, exogenous IL-6 promoted the VEGF-C production of gastric cancer cells and the lymphangiogenesis of HDLECs. As we expected, AG490 was able to reduce these effects. Western blot analysis showed that IL-6 increased JKA, STAT3, p-STAT3 and VEGF-C protein levels in the gastric cancer cells. However, the JKA, STAT3, p-STAT3 and VEGF-C protein expression levels were inhibited by AG490. Our data suggested that IL-6 mediates the singnal pathway of JAK-STAT3-VEGF-C promoting the growth, invasion and lymphangiogenesis in gastric cancer. Thus, IL-6 and its related signal pathways may be a promising target for treatment of gastric cancer growth and lymphangiogenesis.
\end{abstract}

Correspondence to: Professor Jianxin Ye, Department of Gastrointestinal Surgery, First Hospital Affiliated to Fujian Medical University, 20 Cha-Zhong Road, Fuzhou, Fujian 350004, P.R. China E-mail: yejianxinfuyi@126.com

Key words: gastric cancer cell, interleukin-6, proliferation, invasion, JAK-STAT3, VEGF-C

\section{Introduction}

Gastric cancer is a relatively common and second leading cause of cancer death in the world. The Agency for Research on Cancer (IARC) GLOBOCAN reported more than 700,000 deaths of gastric cancer cases and nearly one million new gastric cancer patients occurred globally in 2012 (1). The relative 5-year survival rates for gastric cancer are low in most countries with $<30 \%$ except in South Korea and Japan (2). Gastric cancer has a very poor prognosis due to metastasis. Lymph node metastasis is a major component of gastric cancer metastasis and it is also the most important prognostic factor in patients with gastric cancer. The rate of lymph node metastasis was reported as $20 \%$ even in the early gastric cancer cases (3).

The characteristics of invasive, aggressive and lymph node metastatic gastric cancer are determined by various factors. One of these factors is the tumor microenvironment. Many studies have confirmed that interleukin-6 (IL-6) is a very important cytokine amongst all cytokines of the tumor microenvironment, which promote tumor cell proliferation and invasion. In a previous study, patients with high serum IL-6 levels responded poorly to chemotherapy and was asssociated with an inferior survival outcome in non-small cell lung cancer cases (4). In studies on ovarien cancer, the association between tumor cell growth and the levels of IL- 6 has been demonstrated (5). In addition, they found that high IL-6 expression appears likely to be driven by the ovarian cancer cells (5). In patients with breast carcinoma, it has also been shown that there is a correlation with the serum levels of IL-6 and the degree of malignancy, the extent of tumor invasion, lymph node metastasis and TNM staging (6). Studies have investigated the effcts of IL-6 on tumor cell proliferation, malignant transformation and tumor progression (7).

However, the specific mechanisms responsible for IL-6 promotion of the progression of malignant tumors have not been elucidated. The biological effects of IL-6 are mediated through a membrance receptor complex that contains the receptor of IL-6 (IL-6R) and a regulating receptor gp130. Briefly, IL-6 binds to IL-6R then combines the receptor gp130, 
causing the activation of Janus tyrosine kinase (JAK) (8). Subsequently the signal-transduction pathways are actived, including the signal pathway of JAK-STATs (JAK-signal transducer and transcription activators), the signal pathway of Ras-MAPKs (Ras-mitogen activated protein kinase) and the signal pathway of PI3K-Akt (phosphoninositol-3 kinase-AKT) $(9,10)$. Ara and Declerck $(11)$ reported that the IL- 6 promoting the bone and bone marrow metastasis of various cancer may be via all three signal pathways of JAK-STATs, Ras-MAPKs and PI3K-Akt. Recently, the IL- 6 has been reported as a potent inducer of epithelialmesenchymal transition (EMT) in breast cancer cells may signal through the pathway of JAK-STATs-E-cadherin (12). A very important finding was that the blockade of IL-6R suppressed the proliferation of H460 lung cancer stem cells (13).

Although, previous studies have shown that an increased levels of serum IL-6 was found to be negatively correlated with survival in patients with gastric carcinoma (14), the underlying specific mechanisms remain largely unknown. Lin et al (15) have shown that IL-6 promoted AGS gastric cancer cell invasion via activation of the Src-RhoA-ROCK signaling pathway. Inhibition of the JAK-STAT3 pathway also reduced gastric cancer growth in vitro and in vivo (16). However, the role of IL-6 in modulating the proliferation, invasion and lymph node metastasis is not fully understood.

Therefore, we performed the present study to investigate various gastric cancer cell lines expressing IL-6R and gp130. Exogenous IL-6 stimulation of the gastric cancer cells was used in order to determine the effects of IL- 6 on gastric cancer proliferation, invasion and metastasis, as well as the underlying mechanisms involved.

\section{Materials and methods}

Cell culture. The gastric cancer cell lines SGC-7901, MGC, MKN-28 and AGS were provided by the Key Laboratory of the Ministry of Eduction for Gastrointestinal Cancer, Fujian Medical Universtiy, Fuzhou, Fujian, China. All the cell lines were cultured in RPMI-1640 medium supplemented with $10 \%$ fetal bovine serum (FBS) (both from Gibco, Carlsbad, CA, USA). Human dermal lymphatic endothelial cells (HDLECs, purchased from Sciencell, San Diego, CA, USA) were incubated in endothelial cell medium (Sciencell). All the cells were incubated at $37^{\circ} \mathrm{C}$ under $95 \%$ air and $5 \% \mathrm{CO}_{2}$.

RNA extraction and reverse transcription-polymerase chain reaction $(R T-P C R)$. Total RNA was extracted from the gastric cancer cells grown in 6-well plates using TRIzol reagent (Invitrogen, Carlsbad, CA, USA) according to the manufacturer's. The RNA was quantified by its absorption at $260 \mathrm{~nm}$ and stored at $-80^{\circ} \mathrm{C}$. cDNA was synthesized using the AVM First Strand cDNA synthesis kit (Invitrogen). The primers for IL-6R, gp130 and $\beta$-actin were synthesized according to primer design priciples. The sequences of the primers of IL-6R were: forward, 5'-AGACAGCGTGACTCTGACCT-3' and reverse, 5'-ACTACTGGCGACGCACAT-3' and yielded a 472-bp product. Gp130 yielded a 460-bp product, and the sequences of the primers were as follows: forward, 5'-TCGT GCCTGTTTGCTTGA-3' and reverse, 5'-TGGACTGACGG
AACTTGG-3'; $\beta$-actin yielded a 254 -bp product, as an internal control, and the sequences of the primers were as follows: forward, 5'-CTGTCTGGCGGCACCACCAT-3' and reverse, 5'-GCAACTAAGTCATAGTCCGC-3'. Briefly, RT-PCR was performed under the following conditions: 30 cycles of denaturation at $95^{\circ} \mathrm{C}$ for $30 \mathrm{sec}$, annealing at $55^{\circ} \mathrm{C}$ for $30 \mathrm{sec}$, and extension at $72^{\circ} \mathrm{C}$ for $1 \mathrm{~min}$ followed by $10 \mathrm{~min}$ for final extension at $72^{\circ} \mathrm{C}$. The data of IL-6R and gp130 were normalized relative to the expression of $\beta$-actin mRNA expression in the respective samples.

Western blot analysis. Cells were washed three times with ice cold PBS and then incubated on ice with $250 \mu \mathrm{l}$ of RIPA buffer with $2.5 \mu \mathrm{l}$ phenylmethylsulfonyl fluoride (PMSF) for 15-30 min. The cells were collected and centrifuged at $13,000 \mathrm{rpm}$ for $10 \mathrm{~min}$ at $4^{\circ} \mathrm{C}$. The protein concentrations of cell lysates were measured in duplicate using a BCA protein assay kit (Beyotime Institute of Biotechnology, Shanghai, China). The proportion of protein lysates and 6X loading buffer according to the ratio of $4: 1$ were mixed and then boiled for $5 \mathrm{~min}$ at $100^{\circ} \mathrm{C}$. Equal amounts of total protein were separated on SDS 10\%-PAGE (sodium dodecyl sulfatepolyacrylamide gel electrophoresis) and transferred onto PVDF (polyvinylidene fluoride) membranes. The PVDF membranes were then blocked with $5 \%$ non-fat milk in TBST (Tris-buffered saline with Tween-20) for $2 \mathrm{~h}$ at room temperature. Then, each membrane was incubated with primary antibodies overnight at $4^{\circ} \mathrm{C}$. The diluted primery antibodies, included polyclonal goat anti-human IL-6R antibody (1:500), monoclonal mouse anti-human gp130 (1:500) and $\beta$-actin $(1: 1,500)$ antibody (all from Santa Cruz Biotechnology, Inc. Santa Cruz, CA, USA), the rabbit antihuman VEGF-C antibody (1:500), the goat anti-human p-STAT3 $(1: 1,000)$ (both from Santa Cruz Biotechnology), monoclonal mouse anti-human JAK $(1: 1,000)$, monoclonal mouse anti-human STAT3 (1:1,000) (all from Abcam). After tepeating washing with TBST, the membrane was incubated with secondary antibodies. Using a chemiluminescence western blot immunodetection kit (Invitrogen), we tested immunoreactivity according to the manufacturer's instructions and recorded the data on hyperfine-ECL detection film. The amounts of all protein were semiquantified as ratios to $\beta$-actin on each gel.

Cell proliferation assay. The effects of IL-6 on gastric cell proliferation were assessed using an MTT assay. Cells were seeded in 96-well plates at a density of $2.5 \times 103$ cells/ well and allowed to attach for $12 \mathrm{~h}$. The cells were then treated by different concentrations of IL- 6 (1, 10, 50 and $100 \mathrm{ng} / \mathrm{ml})$ with or without AG490 (50 $\mu \mathrm{mol} / \mathrm{l})$ which was the specific inhibitors of JAK-STAT3 signal pathway for 24,48 and $72 \mathrm{~h}$. Then, the proliferative activity was determined by addition of $10 \mu \mathrm{l}$ of sterile 3-(4,5-dimethylthiazol-2-yl)-2,5-diphenyltetrazolium bromide (MTT) (5 mg/ml; Sigmal, St. Louis, MO, USA) to each well. The reaction was terminated after $4 \mathrm{~h}$ of incubation at $37^{\circ} \mathrm{C}$ by the addition of $100 \mu \mathrm{l}$ of dimethylsulfoxide (DMSO; Sigma). The optical density (OD) value was obtained by measuring absorbance at a wavelength of $570 \mathrm{~nm}$. Each well test was repeated 6 times. 

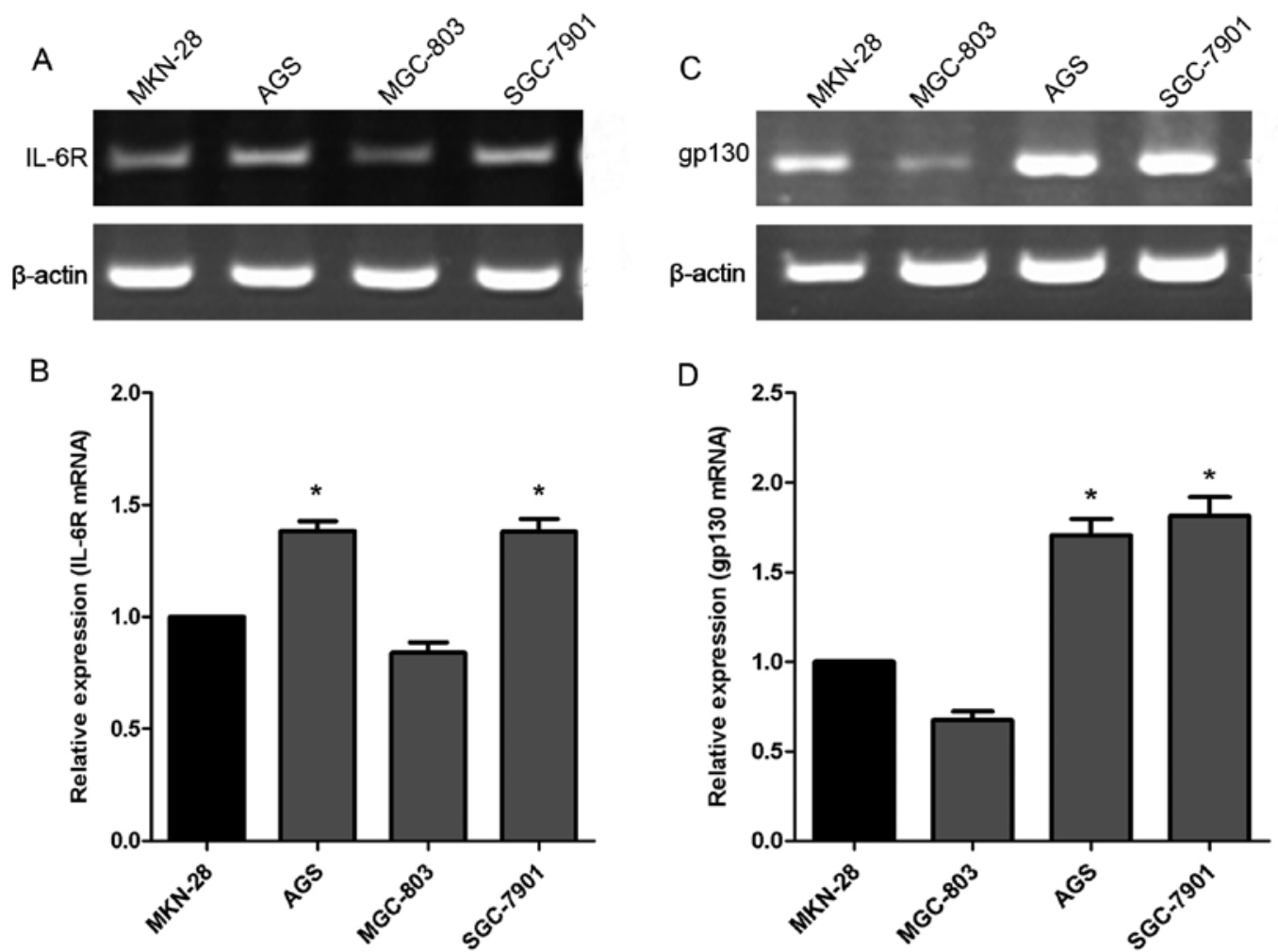

Figure 1. mRNA expression of IL-6R and gp130 in the gastric cancer cell lines SGC-7901, MGC, MKN-28 and AGS cells. (A and B) The IL-6R and gp130 mRNA expression levels in the AGS and SGC-7901 cells was higher than that in the MKN-28 and MGC-803 cells. The mRNA levels of IL-6R and gp130 expression were similar between the AGS and SGC-7901 cells. The mRNA expression of $\beta$-actin was used as an internal control. (B and D) The densitometric value for both groups were normalized to the internal control and relative expression with the following equation: the mRNA expression of IL-6R and gp130 in MKN-28 cells were taken as 1 . Data represent the mean \pm standard deviation (SD), $\mathrm{n}=3 ;{ }^{*} \mathrm{P}<0.05$.

In vitro cell invasion assay. The invasion assay was performed using Transwell 24-well plates with 8- $\mu \mathrm{m}$ polycarbonate membranes (BD Biosciences). Briefly, the upper side of the membranes was coated with Matrigel $(20 \mu \mathrm{g} / \mathrm{well})$ and the membranes were then air-dried for $1 \mathrm{~h}$ of incubation $37^{\circ} \mathrm{C}$. The lower side of the membranes was coated with $5 \mu \mathrm{g}$ fibronectin (BD Biosciences). To test the ability of cell invasion induced by IL- 6 , the treated or untreated gastric cancer cells $\left(2 \times 10^{5}\right)$ in $200 \mu 1$ of RPMI-1640 medium with $2.5 \%$ FBS were placed in the upper chamber. The lower chamber was filled with $700 \mu 1$ RPMI-1640 medium with $10 \%$ FBS as the chemoattractant. The invasion chamber was incubated for $8 \mathrm{~h}$ at $37^{\circ} \mathrm{C}$ and $5 \% \mathrm{CO}_{2}$. The cells on the upper surface of the membrane were removed by gentle scrubbing with a cotton swab. Membranes were fixed in a stationary liquid of $95 \%$ ethanol and $5 \%$ acetic acid for $30 \mathrm{~min}$ and stained with crystal violet. The number of cells on the lower surface of the membrane in 5 random visual fields ( $\mathrm{x} 400$ ) was then counted using a bright field light microscope. Each assay was repeated in triplicate.

Ezyme-liked immunosorbent assay (ELISA). To analyze IL-6 promoting VEGF-C secretion in the gastric cancer cells, the gastric cancer cells were seeded into plates at a density of $3 \times 10^{6}$ cells/well with $4 \mathrm{ml}$ of RPMI-1640 medium supplemented with $10 \%$ FBS. The gastric cancer cells were treated with $100 \mathrm{ng}$ IL-6 or $100 \mathrm{ng}$ IL-6+AG490 $(50 \mu \mathrm{mol} / \mathrm{ml})$ for 24, 48 and $72 \mathrm{~h}$. Using the method of ELISA we tested the amounts of VEGF-C of gastric cancer cell culture supernatants. ELISA was performed using a VEGF-C (H) ELISA kit (Wuhan Boster Biological Technology, Ltd., Wuhan, China). Two hundred microliters of supernatant were added to each well. ELISA was performed according to the manufacturer's instructions. The sensitivity of the assays was $7.8 \mathrm{pg} / \mathrm{ml}$. The absorbance was detected at $450 \mathrm{~nm}$. Each plate test was repeated 3 times.

Human lymphatic capillary endothelial cell (HDLEC) tube-formation assays. To test the gastric cancer cell culture supernatants promoting HDLEC cells the ability of tube-formation, we used the IL-6 (100 ng) and IL-6 (100 ng+AG490) to treat the gastric cancer cells and collected the gastric cancer cell culture supernatants for 24,48 and $72 \mathrm{~h}$. Subsequently, we used the supernatants to culture the HDLECs and observe the role of IL-6 and IL-6+AG490 in the tube formation of HDLECs. The phenomenon of tube formation was observed $1,3,5,8$ and $24 \mathrm{~h}$ after culture. Images were digitally captured at $6 \mathrm{~h}$ after plating. All tube formation experiments were observed by a microscope. Each small tube was counted as 1,1 branch as 2,2 branches as 3, by parity of reasoning. Experiments were repeated 3 times, calculating the average of the tube numbers.

Statistical analysis. Data were analyzed using GraphPad Prism 5 software. Analysis of variance was conducted followed by one-way ANOVA or an unparied t-test. The data 

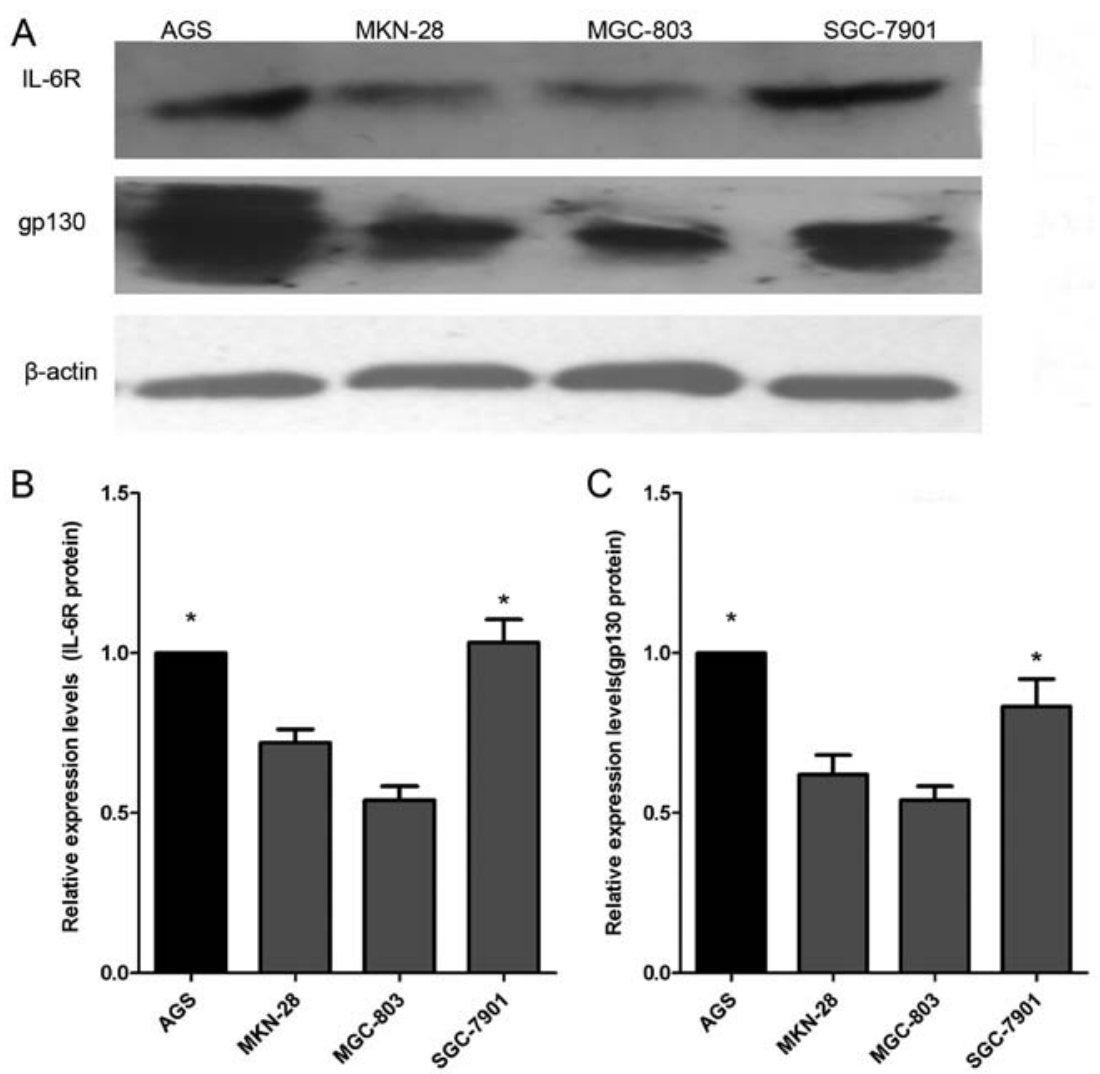

Figure 2. Protein expression of (A) IL-6R and gp130 in the gastric cancer cell lines SGC-7901, MGC, MKN-28 and AGS cells detected by western blot analysis. The IL-6R and gp130 protein expression was semiquantified by western blot analysis. The protein expression of $\beta$-actin was used as an internal control. (B and C) The densitometric value for both groups were normalized to the internal control and relative expression with the following equation: the protein expression of IL-6R and gp130 in AGS cells were taken as 1. Data represent the mean \pm standard deviation (SD), $n=3 ;{ }^{*} \mathrm{P}<0.05$.

are expressed as the means \pm standard deviation (SD). A P-value $<0.05$ was considered to indicate a statistically significant difference.

\section{Results}

mRNA expression of IL-6R and gp130 in gastric cancer cell lines. We analyzed the mRNA expression of IL-6R and gp130 in the gastric cancer cell lines AGS, SGC-7901, MKN-28 and MGC-803. Using RT-PCR, the mRNA expression was detected of IL-6R and gp130 in all gastric cancer cell lines (Fig. 1A and C). The IL-6R and gp130 mRNA expression levels in the AGS and SGC-7901 cells was higher than that in the MKN-28 and MGC-803 cells, and the mRNA levels of IL-6R and gp130 expression were similar between the AGS and SGC-7901 cells (Fig. 1C and D). Thus, we used the AGS and SGC-7901 cells to further examine the role of IL-6.

Protein expression of $I L-6 R$ and gp130 in the gastric cancer cell lines. We tested the IL-6R and gp130 protein expression in the gastric cancer cell lines (AGS, SGC-7901, MKN-28 and MGC-803) by western blot analysis. As expected, the protein expression of IL-6R and gp130 was observed in all cell lines (Fig. 2A). Similarly to mRNA expression of IL-6R and gp130 in the AGS and SGC-7901 cells, the protein expression of IL-6R and gp130 in the AGS and SGC-7901 cells was also higher than that in the MKN-28 and MGC-803 cells (Fig. 2B and C). The protein levels of IL-6R and gp130 expression were similar between the AGS and SGC-7901 cells (Fig. 2B and C). Thus, we used these cells to further examine the role of IL-6.

IL-6 promotes the proliferation of AGS and SGC-7901 cells in vitro. To determine whether the exogenous IL-6 promotes gastric cancer cell proliferation, we first treated the AGS and SGC-7901 cells with different concentrations of IL-6 $(1,10$, 50 and $100 \mathrm{ng} / \mathrm{ml})$ with or without AG490 (50 $\mu \mathrm{mol} / \mathrm{l})$ the specific inhibitors of JAK-STAT3 signal pathway for 24, 48 and $72 \mathrm{~h}$. We examined cell proliferation at 24,48 and $72 \mathrm{~h}$ by MTT assay. When AGS and SGC-7901 cells were treated with increasing concentrations of IL- $6(1,10,50$ and $100 \mathrm{ng} / \mathrm{ml})$, the proliferation increased markedly with the concentration gradient of IL-6. The proliferation of AGS and SGC-7901 cells were significantly increased in a time-dependent manner after treatment with IL-6 (24, 48 and $72 \mathrm{~h}$ ) (Fig. 3A and B). To test the effect of AG490 on the IL-6 increasing proliferation of AGS and SGC-7901 cells, we added the AG490 to the AGS and SGC-7901 cell culture plate to block the JAK-STAT3 signal pathway for 24,48 and $72 \mathrm{~h}$, and then we tested the AGS and SGC-7901 cell proliferation condition by MTT assay. The proliferation effect of IL- 6 was abolished by AG490 treatment (Fig. 3C and D). These results suggested that IL-6 can promote the proliferation of AGS and SGC-7901 cells through the JAK-STAT3 signal pathway.

IL-6 promotes the invasion of the AGS and SGC-7901 cells in vitro. To examine the effect of IL-6 on AGS and SGC-7901 

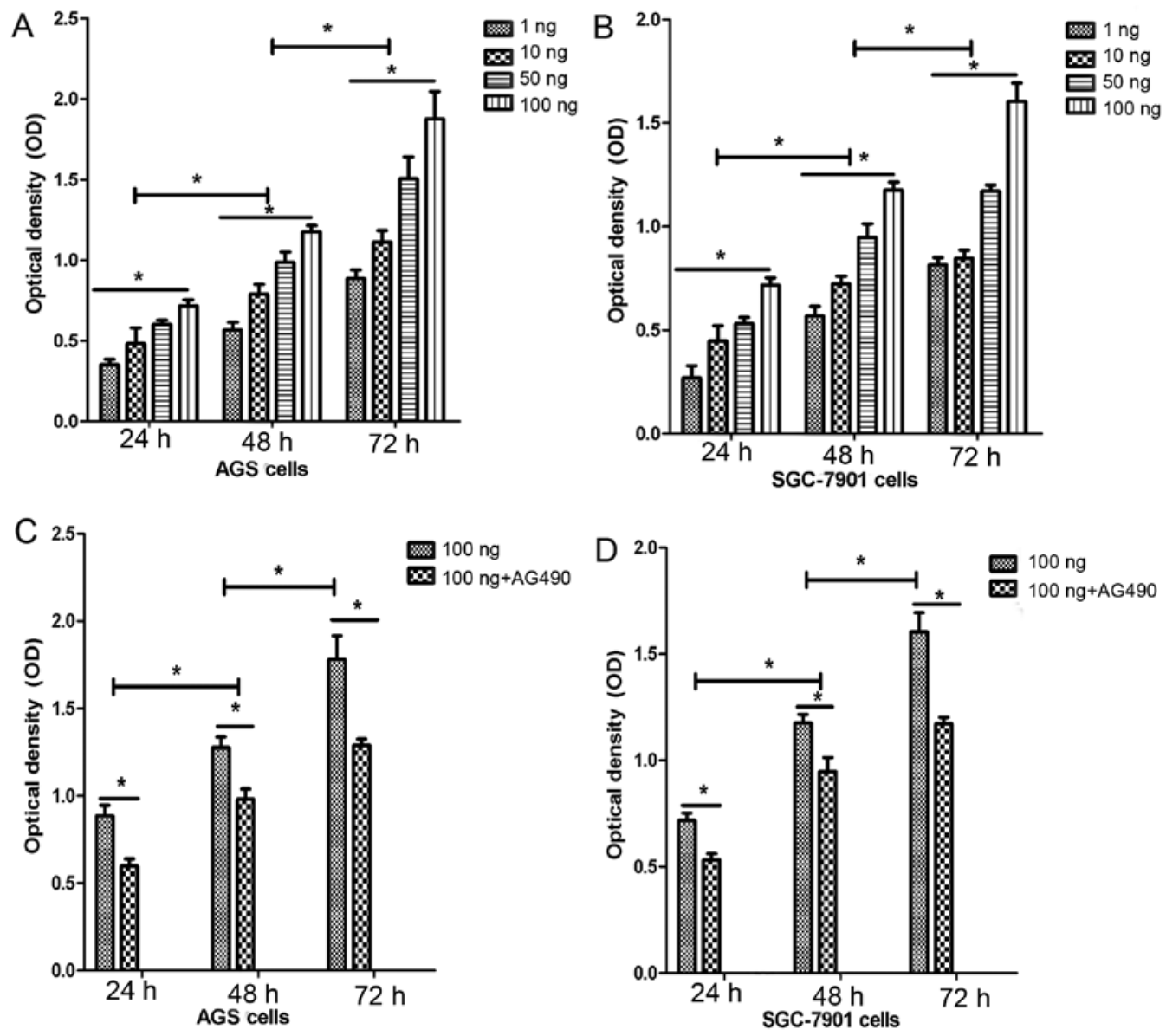

Figure 3. Effects of IL-6 and AG490 which is the specific inhibitor of JAK-STAT3 signal pathway on the proliferation of AGS and SGC-7901 cells. The effects of IL-6 and AG490 on the ability of proliferation of AGS and SGC-7901 cells were analyzed by MTT assay. The proliferation of AGS and SGC-7901 cells were significantly increased in a concentration- and time-dependent manner after IL-6 stimulation (A and B). The proliferative effects of IL-6 were abolished by AG490 treatment in the AGS and SGC-7901 cells (C and D). "P<0.05.

A

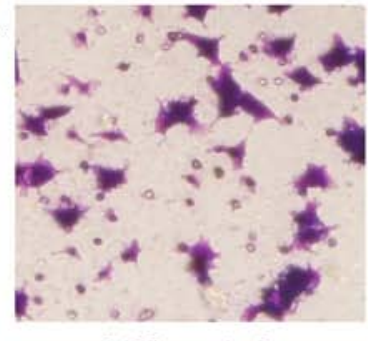

AGS-control

B

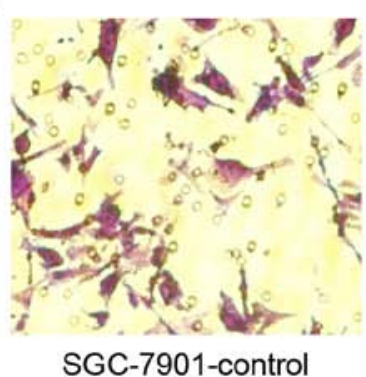

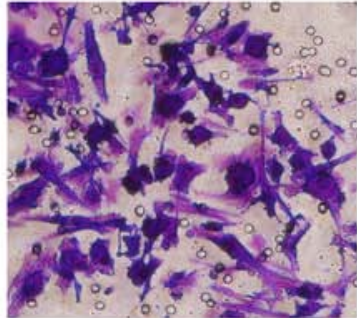

AGS+IL-6

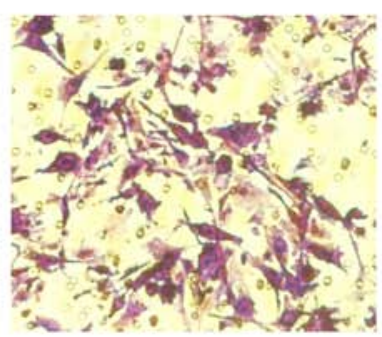

SGC-7901+IL-6

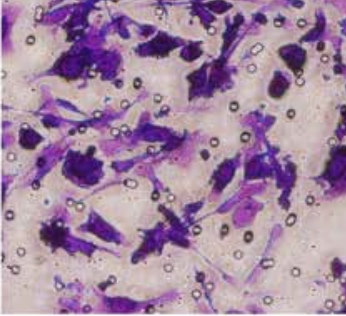

AGS+IL-6+AG490

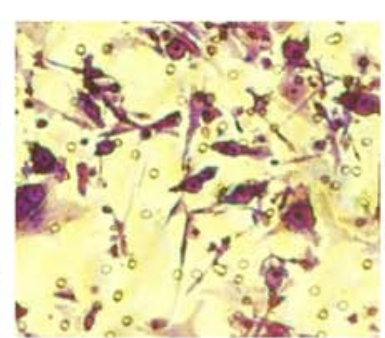

SGC-7901+IL-6+AG490
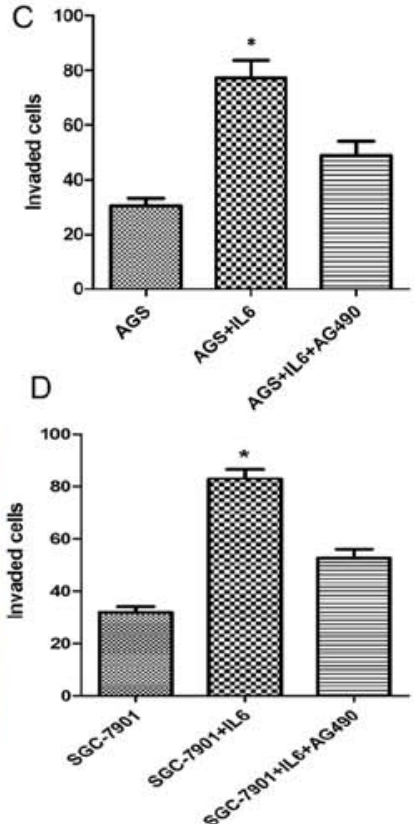

Figure 4. Effects of IL-6 and AG490 which is the specific inhibitor of JAK-STAT3 signal pathway on the invasive ability of AGS and SGC-7901 cells. The effects of IL-6 and AG490 on the invasive ability of AGS and SGC-7901 cells were analyzed by Transwell assay. (A) Invasion ability of AGS cells were significantly increased with IL-6 stimulation. The invasion effects of IL-6 were abolished by AG490 treatment in the AGS cells. (C) Number of invaded cells in AGS-control, AGS+IL-6 and AGS-IL-6+AG490 groups. (B and D) The SGC-7901 cells, and the effects of IL-6 and AG490 on the invasive ability of SGC-7901 is similar to AGS cells (magnification, $\mathrm{x} 400$ ). ${ }^{*} \mathrm{P}<0.05$. 

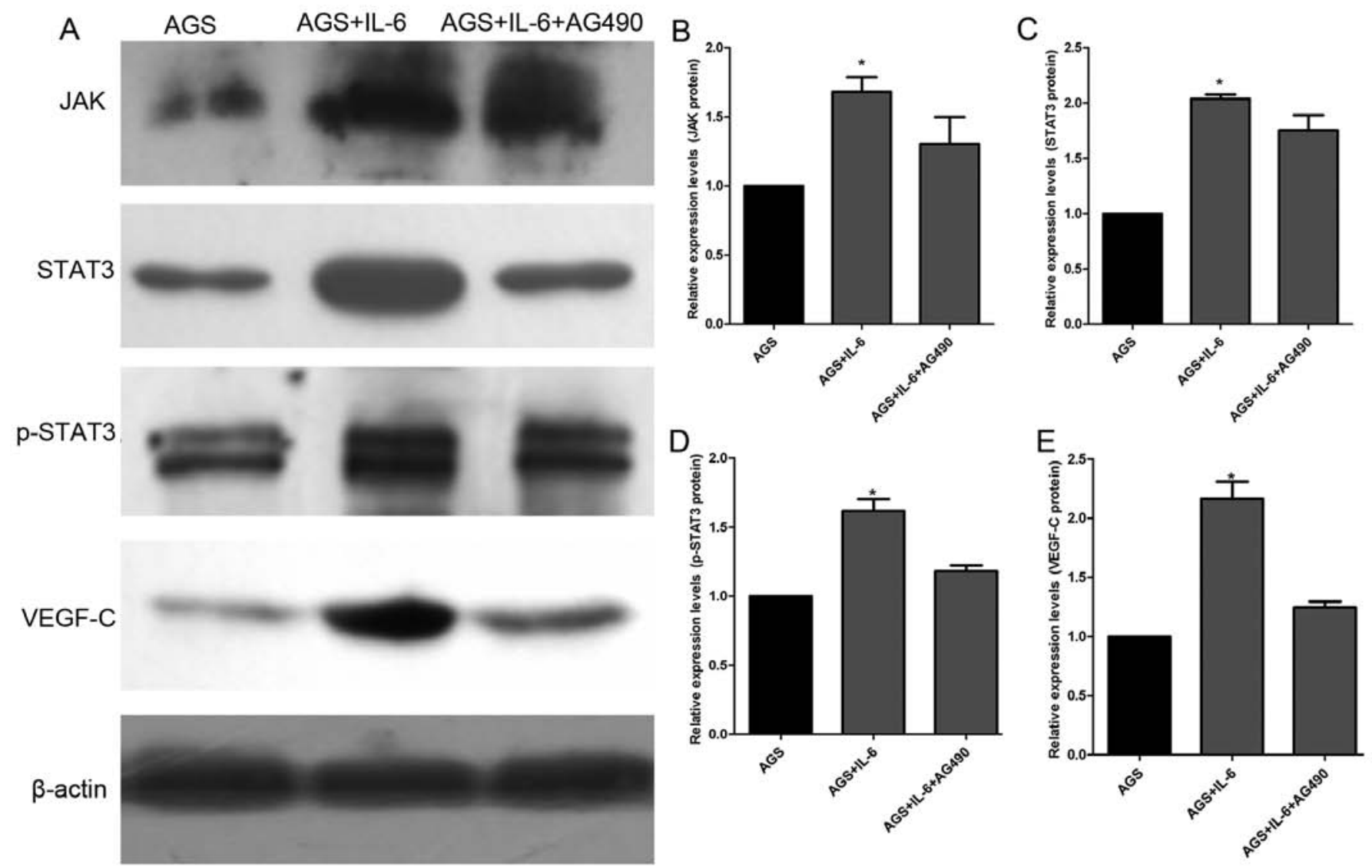

Figure 5. AG490 inhibits the activity of the IL-6-JAK-STAT3-VEGF-C signal pathway. (A) Western blot analysis suggested that the IL-6 markedly promoted the expression of JAK, STAT3, p-STAT3 and VEGF-C protein. The promoting effects of IL- 6 were abolished by AG490 treatment in the AGS cells. $\beta$-actin protein was used as an internal control. The densitometric value for all groups were normalized to the internal control and relative expression with the protein expression of JAK (B), STAT3 (C), p-STAT3 (D) and VEGF-C (E) in AGS group cells were taken as 1. Results are expressed as mean \pm standard deviation (SD), $\mathrm{n}=3 ;{ }^{*} \mathrm{P}<0.05$.

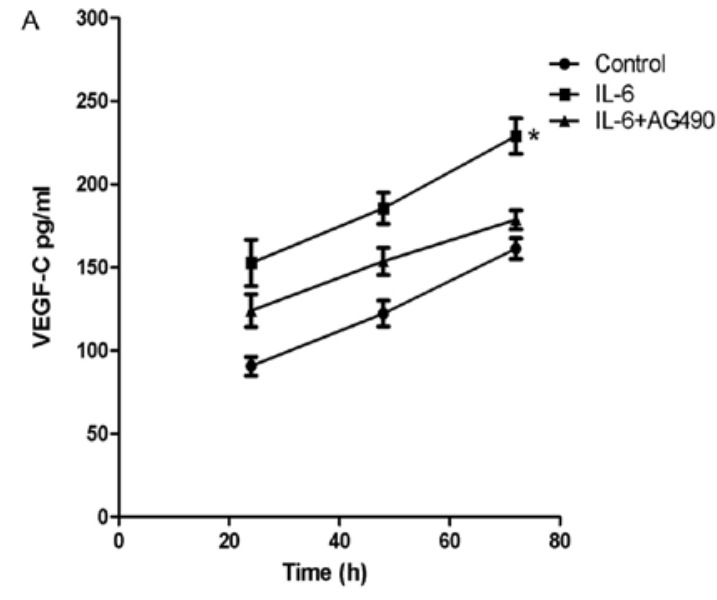

AGS cells

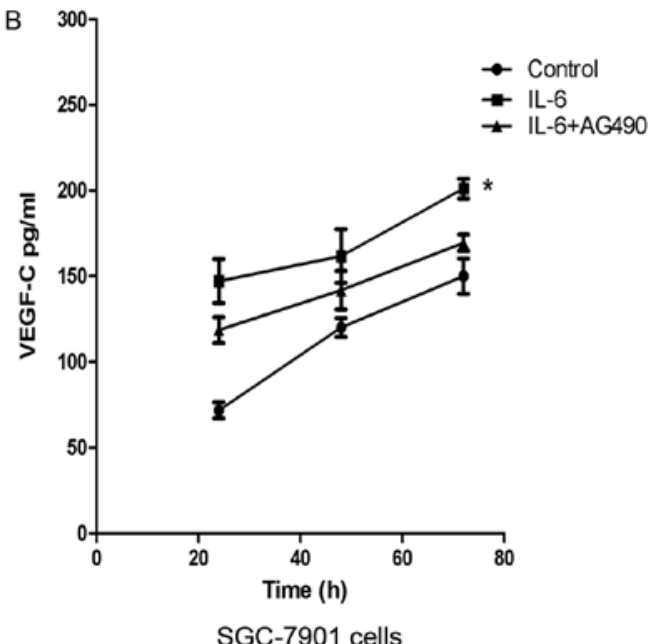

SGC-7901 cells

Figure 6. ELISA analysis of the IL-6 effect of VEGF-C secretion levels in the AGS cells and SGC-7901 cell culture supernatants. The analysis revealed that IL-6 markedly increased VEGF-C secretion levels in the AGS cells and SGC-7901 cell culture supernatants, when compared to the control group (A and B). AG490 significantly suppressed the VEGF-C secretion levels in the AGS and SGC-7901 cell culture supernatants, when compared to the IL-6 group (A and B). ${ }^{*} \mathrm{P}<0.05$.

cell invasion, we used Transwell assay to determine the effects of exogenous IL-6 treated gastric cancer cells. We counted the number of invaded cells. The total number of cells in the
AGS+IL-6 group that invaded through the Transwell polycarbonate filter was significantly higher than that of cells in the AGS group, which was lower than the number of cells in 


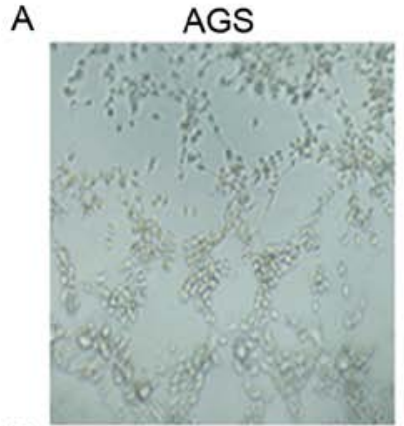

B

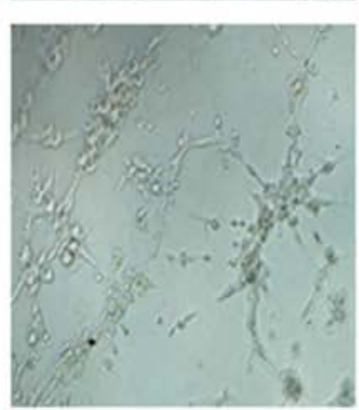

SGC-7901
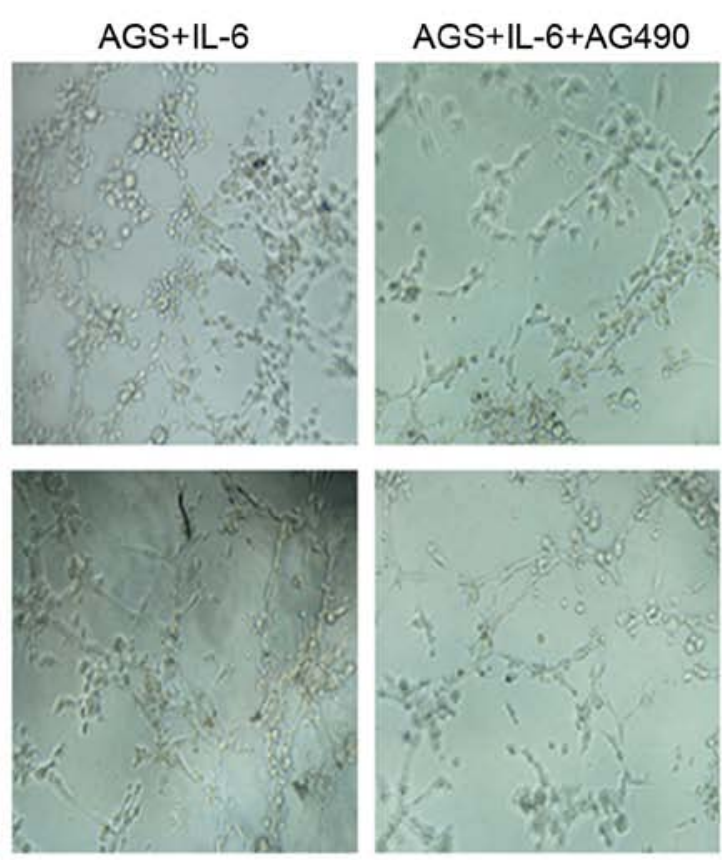

SGC-7901+IL-6

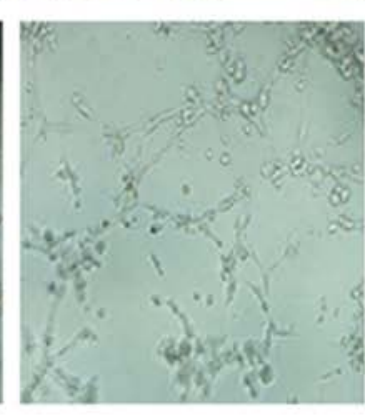

SGC-7901+IL-6+AG490
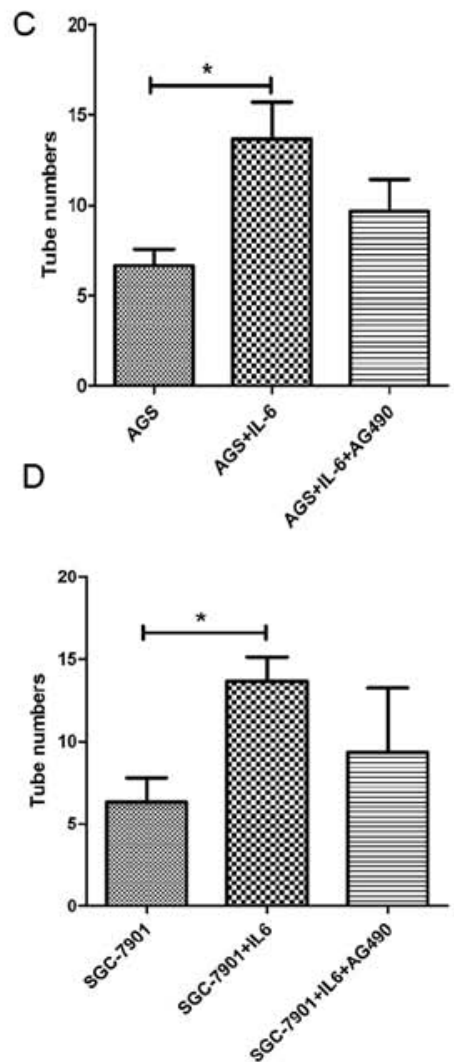

Figure 7. Analysis of the role of IL- 6 in the tube formation of HDLECs. The number of tubes was observed by a microscope for 6 h. In the treatment with IL-6, the tube number of HDLECs increased obviously, adding to the inhibition (AG490) of JAK-STAT3 signal pathway, the tube number of IL-6+AG490 groups were suppressed in the AGS cells and SGC-7901 cells (A and C refer to AGS cells; B and D refer to SGC-7901 cells). (Original magnification, $\mathrm{x} 400) .{ }^{*} \mathrm{P}<0.05$.

the AGS+IL-6+AG490 group (Fig. 4). These data show that the exogenous IL- 6 plays an important role in the invasion of gastric cancer cells.

AG490 inhibits the activity of the IL-6-JAK-STAT3-VEGF-C signal pathway. To investigate the mechanisms of IL-6 responsible for the increase in proliferation and invasion, we assessed the changes in JAK, STAT3, p-STAT3 and VEGF-C levels. The JAK-STAT3 signal pathway is vital to the survival of gastric cancer cells. The western blot analysis results indicated that the JAK, STAT3, p-STAT3 and VEGF-C protein levels in the AGS+IL-6 cells increased in comparison to the AGS+IL-6+AG490 cells, which was higher than the protein levels in the AGS cells (Fig. 5).

IL-6 promotes VEGF-C secretion levels in the AGS cells and $S G C-7901$ cells. We analyzed the IL-6 effect of VEGF-C secretion levels in the AGS cells and SGC-7901 cell culture supernatants. ELISA analysis revealed that IL-6 marked increased VEGF-C secretion levels in the AGS cells and SGC-7901 cell culture supernatants, when compared to the contorl group. Moreover, AG490 significantly suppressed the VEGF-C secretion levels in the AGS cells and SGC-7901 cell culture supernatants, when compared to the IL-6 group (Fig. 6).

IL-6 promotes the tube formation of HDLECs in vitro. After confirming IL-6 induced secretion of VEGF-C in the AGS cells and SGC-7901 cell culture supernatants, we further analyzed the role of the IL- 6 in the tube formation of HDLECs. The number of tubes was observed by a microscope. In the tube formation experiment, the HDLECs cultured had formed a few tubes $2-6 \mathrm{~h}$ after seeding. Thus, we decided to observe the tube formation for $6 \mathrm{~h}$ in our experiment. In the treatment with IL-6, the tube number of HDLECs increased obviously, and adding to the inhibition by AG490 of JAK-STAT3 signal pathway, the tube number of IL-6+AG490 groups were suppressed in the AGS and SGC-7901 cells (Fig. 7).

\section{Discussion}

IL-6 has been described as a factor causing differentiation of B cells to plasma cells, and is an important inflammation cytokine (17). IL- 6 was found to be expressed or produced by various cell types including some tumor cell lines $(18,19)$. IL-6 is a key factor in the growth and differentiation of immune cells and modulate the cell functions, which are involved in the pathogenesis of immune diseases $(20,21)$. It is generally accepted that inflammatory microenvironment plays important roles in tumor development in the tumor growth, invasion and metastasis (22). Although, some studies have shown that IL-6 could promote the progression of gatric cancer and the mechanisms responsible for this phenomenon of tumor evolution (14-16), the underlying specific mechanisms are largely unknown. 
The present study demonstrated the role of exogenous IL-6 in promoting the proliferation, invasion and metastasis of human gastric cancer cells. We further investigated the possible mechanisms underlying this process. We determined whether IL-6R and gp130 were expressed in the gastric cancer cell lines AGS, SGC-7901, MKN-28 and MGC-803. We found the mRNA and protein of IL-6R and gp130 expression in all gastric cancer cell lines, and the mRNA and protein of IL-6R and gp130 expression in the AGS and SGC-7901 cell lines were higher compared to the levels in the MKN-28 and MGC-803 cell lines. The different mRNA and protein of IL-6R and gp130 expression in different gastric cancer cell lines have also been reported by Matsuo et al (23). Thus, we used the AGS and SGC-7901 cells for our exprements.

We examined cell proliferation at 24,48 and $72 \mathrm{~h}$ by MTT assay after IL- 6 was added to the gastric cancer cell culture plate. Compared with the Control and IL-6+AG490 groups, cell proliferation in the IL- 6 group was more rapid, and the cell proliferation in the IL-6+AG490 group was slower than that in the IL-6 group. The data from the in vitro cell proliferation assay indicated that the growth of the gastric cancer cells in the IL- 6 group was markedly increased compared with control group. The AG490, which is the specific inhibitor of the JAK-STAT3 signal pathway, can partly eliminate the promotion effect of cell proliferation function of IL-6 in the AGS and SGC-7901 cells. This was similar to the gastric cancer cell invasion in the AGS and SGC-7901 cells. These results are consistent with previous studies, in which the authors concluded that IL-6 plays an important role in various tumor cell proliferation and invasion (24). Hodge et al (25) reported that IL-6 as a pleiotropic cytokine, promoted the proliferation and invasion of tumor cells. Tchirkov et al (26) showed that IL-6 accelerated the growth of glioblastoma cells and shortened survival in glioblastoma patients. Gao et al (27) also found that IL-6 promoted lung cancer cell proliferation and invasion.

Many studies show that IL-6 promotes tumor cell proliferation, growth $(25,27,28)$ and angiogenesis $(29,30)$, and the JAK-STAT3 signal pathway was the key in the tumor progression (25,27-30). However, it was unknown whether the IL-6 promoted tumor lymphangiogenesis, thereby accelarating the lymp node metastasis of tumor cells. Thus, we analyzed the relationship of the gastric cancer lymp node and the expression levels of IL-6 in the gastric cancer tissues, and found that the expression levels of IL-6 in the gastric cancer tissues and lymp node metastasis were positively correlated (data not shown). This is consistent with a recent study demonstrating that the expression levels of IL-6 is associated with lymph node metastasis rate in the oral squamous cell carcinoma (31). In addition, vascular endothelial growth factor-C (VEGF-C) is an important factor of the VEGF family and has been correlated with lymphatic metastasis in many carcinomas, including gastric cancer (32), moreover, VEGF-C is an important factor, which promotes lymphangiogenesis (33).

In view of the foregoing, we speculated that IL- 6 could promote VEGF-C production in gastric cancer cells and the JAK-STAT3 plays an important role in the process. In this study, we assessed the changes in JKA, STAT3, p-STAT3 and VEGF-C protein expression. The JAK-STAT3 signal pathway and VEGF-C are vital to the survival of cancer cells $(25,27,34)$. The western blot analysis indicated that JKA, STAT3, p-STAT3 and VEGF-C protein levels in the AGS+IL-6 cells were increased compared with those in the AGS cells. However, the JKA, STAT3, p-STAT3 and VEGF-C protein expression levels were inhibited in the AGS+IL-6+AG490 cells. In the present study, to determine whether the promoting effects of IL- 6 affected the VEGF-C production in the AGS cells and SGC-7901 cell culture supernatants, we employed the method of ELISA to test the VEGF-C production levels in the AGS cells and SGC-7901 cell culture supernatants. The results shown that IL-6 promoted the VEGF-C production and the AG490 inhibited the promotive effects of IL-6 in the AGS and SGC-7901 cells. We then further analyzed the role of the IL-6 in the tube formation of HDLECs, in which the function of VEGF-C is very important (35). In the treatment with IL-6, the tube number of HDLECs increased obviously, and by adding the inhibitor AG490 of JAK-STAT3 signal pathway, the tube number of IL-6+AG490 groups were suppressed in the AGS and SGC-7901 cells. These results suggest that IL-6 can promot the VEGF-C production via the JAK-STAT3 signal pathway.

In the present study, we verified the biological functions of IL-6 on various gastric cancer cell lines. We provided evidence that IL- 6 can promote the gastric cancer cell proliferation, invasion and lymphangiogenesis in vitro and further found that the underlying mechanisms may be via the JAK-STAT3-VEGF-C signal pathway. Collectively the above suggests that IL-6 is involved in the progression of gastric cancer and represents a potential molecular target in the treatment of gastric cancer.

\section{Acknowledgements}

The present study was supported by the Key Project of Science and Technology Reserch Program in Fujian Province (no. 2012B002), the Fujian Provincial Natrual Science Foundation (no. 2014J01309), the Backbone Teacher Project of Fujian Medical University (no. JGG200716), the China Non intervention Gastric Cancer Registration Survey Clinical Research Projects (no. QT-201403), the Ministry of Health Medicine Science and Technology Development and Research (no. W2013FZ08) and the National Clinical Key Specialty Construction Project (General Surgery) of China.

\section{References}

1. Ferlay J,Soerjomataram I, Ervik M, Dikshit R, Eser S, Mathers C, Rebelo M, Parkin DM, Forman D and Bray F: GLOBOCAN 2012v1.0, Cancer incidence and mortality worldwide: IARC CancerBase No. 11 [internet]. International Agency for Research on Cancer, Lyon, 2013 [cited 2014 Apr 25]. Available from: http:// globocan.iarc.fr.

2. Carneiro F: Stomach cancer. In: World Cancer Report 2014. Steward B and Wild CP (eds). International Agency for Research on Cancer, Lyon, pp383-391, 2014.

3. Koufuji K, Takeda J, Toyonaga A, Yoshihara S, Tanaka Y, Ohta J, Aoyagi K, Yano S, Kodama I and Shirouzu K: Early gastric cancer and lymph node metastasis. Kurume Med J 44: 157-164, 1997.

4. Chang CH, Hsiao CF, Yeh YM, Chang GC, Tsai YH, Chen YM, Huang MS, Chen HL, Li YJ, Yang PC, et al: Circulating interleukin-6 level is a prognostic marker for survival in advanced nonsmall cell lung cancer patients treated with chemotherapy. Int J Cancer 132: 1977-1985, 2013. 
5. Yanaihara N, Anglesio MS, Ochiai K, Hirata Y, Saito M, Nagata C, Iida Y, Takakura S, Yamada K, Tanaka T, et al: Cytokine gene expression signature in ovarian clear cell carcinoma. Int J Oncol 41: 1094-1100, 2012.

6. Ravishankaran P and Karunanithi R: Clinical significance of preoperative serum interleukin- 6 and $\mathrm{C}$-reactive protein level in breast cancer patients. World J Surg Oncol 9: 18, 2011.

7. Trikha M, Corringham R, Klein B and Rossi JF: Targeted anti-interleukin-6 monoclonal antibody therapy for cancer: a review of the rationale and clinical evidence. Clin Cancer Res 9: 4653-4665, 2003.

8. Jones SA, Horiuchi S, Topley N, Yamamoto N and Fuller GM: The soluble interleukin 6 receptor: mechanisms of production and implications in disease. FASEB J 15: 43-58, 2001.

9. Grivennikov S and Karin M: Autocrine IL-6 signaling: A key event in tumorigenesis? Cancer Cell 13: 7-9, 2008.

10. Heinrich PC, Behrmann I, Haan S, Hermanns HM, MüllerNewen G and Schaper F: Principles of interleukin (IL)-6-type cytokine signalling and its regulation. Biochem J 374: 1-20, 2003.

11. Ara T and Declerck YA: Interleukin- 6 in bone metastasis and cancer progression. Eur J Cancer 46: 1223-1231, 2010.

12. Xie G, Yao Q, Liu Y, Du S, Liu A, Guo Z, Sun A, Ruan J, Chen L, Ye C, et al: IL-6-induced epithelial-mesenchymal transition promotes the generation of breast cancer stem-like cells analogous to mammosphere cultures. Int J Oncol 40: 1171-1179, 2012.

13. Yi H, Cho HJ, Cho SM, Jo K, Park JA, Kim NH, Amidon GL, Kim JS and Shin HC: Blockade of interleukin-6 receptor suppresses the proliferation of $\mathrm{H} 460$ lung cancer stem cells. Int J Oncol 41: 310-316, 2012.

14. Liao WC, Lin JT, Wu CY, Huang SP, Lin MT, Wu AS, Huang YJ and Wu MS: Serum interleukin-6 level but not genotype predicts survival after resection in stages II and III gastric carcinoma. Clin Cancer Res 14: 428-434, 2008.

15. Lin MT, Lin BR, Chang CC, Chu CY, Su HJ, Chen ST, Jeng YM and Kuo ML: IL-6 induces AGS gastric cancer cell invasion via activation of the c-Src/RhoA/ROCK signaling pathway. Int J Cancer 120: 2600-2608, 2007.

16. Judd LM, Menheniott TR, Ling H, Jackson CB, Howlett M, Kalantzis A, Priebe W and Giraud AS: Inhibition of the JAK2/ STAT3 pathway reduces gastric cancer growth in vitro and in vivo. PLoS One 9: e95993, 2014.

17. Hirano T, Taga T, Nakano N, Yasukawa K, Kashiwamura S, Shimizu K, Nakajima K, Pyun KH and Kishimoto T: Purification to homogeneity and characterization of human B-cell differentiation factor (BCDF or BSFp-2). Proc Natl Acad Sci USA 82: $5490-5494,1985$.

18. Grossman RM, Krueger J, Yourish D, Granelli-Piperno A, Murphy DP, May LT, Kupper TS, Sehgal PB and Gottlieb AB: Interleukin 6 is expressed in high levels in psoriatic skin and stimulates proliferation of cultured human keratinocytes. Proc Natl Acad Sci USA 86: 6367-6371, 1989.

19. Kishimoto T: The biology of interleukin-6. Blood 74: 1-10, 1989.

20. Williams N, De Giorgio T, Banu N, Withy R, Hirano T and Kishimoto T: Recombinant interleukin 6 stimulates immature murine megakaryocytes. Exp Hematol 18: 69-72, 1990.

21. Hirano T, Matsuda T, Turner M, Miyasaka N, Buchan G, Tang B, Sato K, Shimizu M, Maini R, Feldmann M, et al: Excessive production of interleukin 6/B cell stimulatory factor-2 in rheumatoid arthritis. Eur J Immunol 18: 1797-1801, 1988.
22. Grivennikov SI, Greten FR and Karin M: Immunity, inflammation, and cancer. Cell 140: 883-899, 2010.

23. Matsuo K, Oka M, Murase K, Soda H, Isomoto H, Takeshima F, Mizuta Y, Murata I and Kohno S: Expression of interleukin 6 and its receptor in human gastric and colorectal cancers. J Int Med Res 31: 69-75, 2003.

24. Ataie-Kachoie P, Pourgholami MH and Morris DL: Inhibition of the IL-6 signaling pathway: A strategy to combat chronic inflammatory diseases and cancer. Cytokine Growth Factor Rev 24: 163-173, 2013.

25. Hodge DR, Hurt EM and Farrar WL: The role of IL-6 and STAT3 in inflammation and cancer. Eur J Cancer 41: 2502-2512, 2005.

26. Tchirkov A, Khalil T, Chautard E, Mokhtari K, Véronèse L, Irthum B, Vago P, Kémény JL and Verrelle P: Interleukin-6 gene amplification and shortened survival in glioblastoma patients. $\mathrm{Br}$ J Cancer 96: 474-476, 2007.

27. Gao SP, Mark KG, Leslie K, Pao W, Motoi N, Gerald WL, Travis WD, Bornmann W, Veach D, Clarkson B, et al: Mutations in the EGFR kinase domain mediate STAT3 activation via IL-6 production in human lung adenocarcinomas. J Clin Invest 117: 3846-3856, 2007.

28. Kudo M, Jono H, Shinriki S, Yano S, Nakamura H, Makino K, Hide T, Muta D, Ueda M, Ota K, et al: Antitumor effect of humanized anti-interleukin-6 receptor antibody (tocilizumab) on glioma cell proliferation. Laboratory investigation. J Neurosurg 111: 219-225, 2009.

29. Shinriki S, Jono H, Ota K, Ueda M, Kudo M, Ota T, Oike Y, Endo M, Ibusuki M, Hiraki A, et al: Humanized anti-interleukin-6 receptor antibody suppresses tumor angiogenesis and in vivo growth of human oral squamous cell carcinoma. Clin Cancer Res 15: 5426-5434, 2009.

30. Huang SP, Wu MS, Wang HP, Yang CS, Kuo ML and Lin JT: Correlation between serum levels of interleukin-6 and vascular endothelial growth factor in gastric carcinoma. J Gastroenterol Hepatol 17: 1165-1169, 2002.

31. Jinno T, Kawano S, Maruse Y, Matsubara R, Goto Y, Sakamoto T, Hashiguchi Y, Kaneko N, Tanaka H, Kitamura R, et al: Increased expression of interleukin- 6 predicts poor response to chemoradiotherapy and unfavorable prognosis in oral squamous cell carcinoma. Oncol Rep 33: 2161-2168, 2015.

32. Jüttner S, Wissmann C, Jöns T, Vieth M, Hertel J, Gretschel S, Schlag PM, Kemmner W and Höcker M: Vascular endothelial growth factor-D and its receptor VEGFR-3: Two novel independent prognostic markers in gastric adenocarcinoma. J Clin Oncol 24: 228-240, 2006.

33. Mandriota SJ, Jussila L, Jeltsch M, Compagni A, Baetens D, Prevo R, Banerji S, Huarte J, Montesano R, Jackson DG, et al: Vascular endothelial growth factor-C-mediated lymphangiogenesis promotes tumour metastasis. EMBO J 20: 672-682, 2001

34. Chen Y, Jiang L, She F, Tang N, Wang X, Li X, Han S and Zhu J: Vascular endothelial growth factor-C promotes the growth and invasion of gallbladder cancer via an autocrine mechanism. Mol Cell Biochem 345: 77-89, 2010.

35. Du Q, Jiang L, Wang X, Wang M, She F and Chen Y: Tumor necrosis factor- $\alpha$ promotes the lymphangiogenesis of gallbladder carcinoma through nuclear factor- $\mathrm{KB}$-mediated upregulation of vascular endothelial growth factor-C. Cancer Sci 105: 1261-1271, 2014. 